\title{
FISSIDENTETUM TAXIFOLII WALDHEIM 1944 RHIZOMNIETOSUM PUNCTATI SUBAS. NOVA EN EL PISO SUPRAMEDITERRANEO DE SIERRA NEVADA (GRANADA, ESPAÑA)
}

\author{
J. A. GIL \& F. MARTINEZ
}

RESUMEN: La asociación Fissidentetun taxifolii Waldheim 1944 rhizonietosum punctati subas. nova, ha sido observada en taludes húmedos del piso supramediterráneo de Sierra Nevada. Aparece ligada al bioclima de formaciones boscosas de Quercus pyrenaica Willd., mostrando una posición finícola y relíctica en el sur de Europa.

RESUME: L'association Fissidentetun taxifolii Waldheim 1944 rhizomnietosum punctati subas. nova, est observée a Sierra Nevada dans les talus humides de l'étage supraméditerranéen; elle est liée au bioclimat des formations forestiers du Quercus pyrenaica Willd., montrant une position finicole et rélictique.

\section{INTRODUCCION}

El piso de vegetación supramediterráneo de Sierra Nevada, comprendido entre $1000 \mathrm{~m}$ (vertiente sur) y $2000 \mathrm{~m}$, se caracteriza por una temperatura media anual (T) de $11,8{ }^{\circ} \mathrm{C}$, una temperatura media de las mínimas del mes más frío (m) de $-2{ }^{\circ} \mathrm{C}$ a $1500 \mathrm{~m}$ y $-2,99{ }^{\circ} \mathrm{C}$ a $1750 \mathrm{~m}$. La precipitación media anual (P) es de 692,5 $\mathrm{mm}$ correspondiente a un ombroclima subhúmedo. En este bioclima, la vegetación climácica se configura en un melojar de Adenocarpo-Quercetum pyrenaicae Martínez Parras \& Molero Mesa 1982, perteneciente al dominio de Quercion robori-pyrenaicae (Br.-Bl. \& al. 1956) RivasMartínez 1974, cuyo carácter residual determina que, en general, aparezca degradado en sus etapas de sustitución de espinales de Lonicero-Berberidion hispanicae Bolós 1954 y retamares de Cytision scopariae Tx. apud Preising 1949.

Los suelos en que se asientan estas formaciones se desarrollan sobre micaesquistos y cuarcitas, estando constituidos por un complejo formado por cambisoles y regosoles eútricos en las zonas de degradación, enriquecidos por un phaeozem háplico en las zonas mejor conservadas que posee un horizonte A orgánico-mineral, saturado en bases en más del 50\% (epipedón móllico).

En el seno de este hábitat, ha sido reconocida una comunidad briofítica terricola de Fissidens taxifolius y Rhizomnium punctatum que asimilamos a la Fissidentetum taxifolii Waldheim 1944, representada a través de una nueva subasociación rhizomnietosum punctati que describimos a continuación.

La nomenclatura adoptada es la de Casas (1981) para losmusgos y paralas hepáticas se ha seguido a Grolle(1976). 


\section{ESTUDIO BRIOSOCIOLOGICO}

Fissidentetum taxifolii Waldheim 1944 rhizomnietosum punctati subas. nova

Síntipo: Tabla 1, invent. 1-30

Holosintipo: Invent. 8

\section{Especie diferencial: Rhizomnium punctatum}

Número medio de especie: 6,9. Cobertura media: 74,6. Superficie media de los inventarios: 20,2 .

Sinecología: La comunidad aparece siempre en microclimas de gran humedad edáfica, compensatoria de la xericidad ambiental, por lo que halla refugio en vaguadas y barrancos del piso supramediterráneo. Los protosuelos arcillosos-limosos y humificados son su hábitat más frecuente, desarrollados sobre esquistos húmedos a rezumantes, en ocasiones expuestos a una leve y temporal escorrentía, pues es normal su localización en inmediaciones de manantiales y cascadas.

El despoblamiento forestal que caracteriza a gran parte del piso supramediterráneo nevadense, además de un ombroclima subhúmedo con tendencia a hacerse seco en determinadas zonas, impide el desarrollo de la asociación en estaciones desprotegidas. Es por ello que en su' límite de área, la comunidad adopta un comportamiento microclimático finicola.

En cuanto a su comportamiento edáfico. la subasociación rhizomnietosum punctati que proponemos admite un amplio margen de $\mathrm{pH}$, pues coloniza sustratos neutros como débilmente ácidos ( $\mathrm{pH}:$ 6,37,3), a veces con un importante contenido en bases, por lo que de acuerdo con las precisiones de Apinis \& Lacis (1936) la consideramos hipoacidoneutrófila (intérvalo de $\mathrm{pH}$ entre 5,6 y 7,1). Asimismo puede calificarse de esciófila, terri-saxi-humícola y mesohigrófila. Por otra parte, las comunidades de Fissidens taxifolius descritas, destacan por la variabilidad de los sustratos en que crecían (cfr. Haybach, 1956: 159; Philippi, 1965: 654; Dünk, 1972: 41; Neumayr, 1971: 124; Hübschmann, 1975: 304), si bien Marstaller (1980:99) precisa que Fissidentetum taxifolii debe considerarse neutrófila.

Sinfisionomía: La asociación forma tapices relativamente cerrados $(74,6 \%)$ que en la variante típica de Amphidium mougeottii se reducen un tanto $(68,4 \%)$, mientras que en la variante de Plagiomnium undulatum aumenta hasta hacerse cerrada (81,3\%). Estos tapices tienen un espesor medio de $3 \mathrm{~cm}$, que en la variante de Plagiomnium undulatum alcanzan $5 \mathrm{~cm}$.

La comunidad se estructura con elementos vivaces, totalmente dominantes, siendo los más caracterizados los de biótipo Bryochamaephyta y Bryochamaephyta caespitosa (Fissidens taxifolius, Rhizomnium punctatum, Bryum pseudotriquetrum); las restantes poseen biótipo Bryochamaephyta reptantia que contrasta con las anteriores. Esta estructura se altera en la variante de Plagiomnium undulatum, pues su desarrollo estolonífero oculta al resto de los integrantes de la comunidad que aparece biestratificada. En grado menor, la variante de Amphidium mougeotti tambien manifiesta diferencias fisionómicas respecto a la típica, en base a que la especie diferencial es de biótipo intermedio entre las Bryochamaephyta caespitosa y pulvinata (Ochsner, 1975: 234). 
Composición florística: La subasociación rhizomnietosum punctati está dominada por elementos circumpolares $(71,7 \%)$, así Fissidens taxifolius (V), Rhizomnium punctatum (V), Eurhynchium praelongum (IV), Bryum pseudotriquetrum (III), Chiloscyphus polyanthos (III) y Pellia epiphylla (II), seguidas a distancia de los circumpolares montanos $(10,8 \%)$, circumpolares atlánticos $(6,6 \%)$, mediterráneo-atlánticos $(6,3 \%)$ y cosmopolitas $(4,6 \%)$. Por sus afinidades edáficas, está integrada por especies que son fundamentalmente indiferentes a la reacción del sustrato $(70,2 \%)$, acompañadas de algunas de afinidad basófila $(16,8 \%)$ y acidófila $(13 \%)$. Las modificaciones que se producen de tipo biogeográfico y edáficas en las distintas variantes de la asociación se reflejan en los cuadros 1 y 2 .

\begin{tabular}{|c|c|c|c|c|c|}
\hline & CIRCUMPOLARES & \begin{tabular}{|l|} 
CIRCUMPOLARES \\
MONTANAS
\end{tabular} & \begin{tabular}{|l} 
CIRCUMPOLARES \\
ATLANTICAS
\end{tabular} & $\begin{array}{l}\text { MEDI TERRANEO } \\
\text { ATLANTICAS }\end{array}$ & COSMOPOLITAS \\
\hline typicum & $75 \%$ & $16,7 \%$ & 0 & 0 & $8,3 \%$ \\
\hline $\begin{array}{l}\text { var. de Amphidium } \\
\text { undulatum }\end{array}$ & $75 \%$ & $16,7 \%$ & 0 & $8,3 \%$ & 0 \\
\hline $\begin{array}{l}\text { var. de Plagiomnium } \\
\text { undulatum }\end{array}$ & $70 \%$ & $10 \%$ & $10 \%$ & 0 & $10 \%$ \\
\hline $\begin{array}{l}\text { Facies de Pellia } \\
\text { endiviifolia y Thamno- } \\
\text { bryum alopecurum }\end{array}$ & $68,8 \%$ & 0 & $16,6 \%$ & $16,6 \%$ & 0 \\
\hline TOTAL & $71,7 \%$ & $10,8 \%$ & $6,6 \%$ & $6,3 \%$ & $4,6 \%$ \\
\hline
\end{tabular}

Cuadro 1.- Espectro biogeográfico.

\begin{tabular}{|l|c|c|c|}
\cline { 2 - 4 } \multicolumn{1}{l|}{} & INDIFERENTES & ACIDOFILAS & BASOF ILAS \\
\hline typicum & $66 \%$ & 16,7 & $16,7 \%$ \\
\hline $\begin{array}{l}\text { var. de Amphidium } \\
\text { mougeottii }\end{array}$ & $50,3 \%$ & $25 \%$ & $16,7 \%$ \\
\hline $\begin{array}{l}\text { var. de Plagiomnium } \\
\text { undulatum }\end{array}$ & $66,7 \%$ & 0 & 0 \\
\hline $\begin{array}{l}\text { facies de Pellia } \\
\text { endiviifolia y Thamno- } \\
\text { bryum alopecurum }\end{array}$ & $70,2 \%$ & $13 \%$ & $33,3 \%$ \\
\hline I0IAL & $90 \%, 8 \%$ \\
\hline
\end{tabular}


Sindinámica: El estadio inicial corresponde a las especies terri-saxícolas, Fissidens taxifolius y Eurhynchium praelongum, característica y diferencial territorial de la asociación, respectivamente. Dado que ésta se establece en microclimas húmedos de suelos rezumantes, se incorporan especies que refrendan la tendencia mesohigrófila de la comunidad en Sierra Nevada; en este sentido, destaca de modo especial Rhizomnium punctatum como diferencial de la subasociación que se describe, acompañándole otras de comportamiento helofítico como Pellia epiphylla, Philonotis fontana, Bryum pseudotriquetrum, Chiloscyphus polyanthos y Brachythecium rivulare. En ocasiones la humificación del suelo es notable, siendo Plagiomnium undulatum quien se agrega a las anteriores además de Cardamine sylvatica. Todo ello expresa una transición hacia comunidades esciófilas, helo-higrófilas y terri-humicolas de la subalianza Brachythecio-Cardaminenion Mas 1959 (a través de las asociaciones Pellio-Plagiomnietum rostratae Varo \& Gil 1982 y Bryo-Plagiomnietum undulatae Varo \& Gil 1982), de la clase MontioCardaminetea Br.-Bl. \& Tx. 1943, por lo que es frecuente hallarlas en ecótono formando complejos.

Variabilidad: Para la subasociación rhizomnietosum punctati reconocemos la variante típica o de Rhizomnium punctatum y las variantes de Amphidium mougeottii y de Plagiomnium undulatum.

Variante typicum o de Rhizomnium punctatum (tabl.1, invent. $1-13)$

Es la más frecuente pero de mayor probreza florística, coincidiendo con el estadio pionero en taludes húmedos y de escasa profundidad de suelo. Se diferencia ecológicamente por ser terri-saxícola, en ocasiones de carácter exocomofítico. Suele hallarse en contacto con las variantes de Amphidium mougeottii y Plagiomnium undulatum. Sintaxonómicamente está relacionada con Fissidens taxifolius-Eurhynchium speciosum-Verein Haybach 1956: 159, tabl.5, p.p.

Variante de Amphidium mougeottii (tabl.1, invent. 14-19)

Variante orófila y casmocomofítica, en la que Amphidium mougeottii actua como pionera de fisuras rezumantes, expuestas o no, acumulando un protosuelo que es invadido por las restantes especies de la comunidad, hasta erigirse en la variante de mayor riqueza florística. Como la variante típica es mesohigrófila y terri-saxicola, pero foto-esciófila y exclusivamente orófila. Parece estar relacionada sintaxonómicamente con Amphidium mougeottii-Ass. Stфomer 1939 sensu Nörr (1969: 387, tabl.12, p.p. min.) y con Blindio-Amphoridietum Hébrard 1973: 354, tabl. 2. p.p. min.

Variante de Plagiomnium undulatum (tabl.1, invent. 20-30)

Representa el estadio más evolucionado, logrando la máxima colonización y cobertura $(81,3 \%)$. Su presencia está ligada a taludes con suelos profundos y consolidados, muy húmedos y humificados; se diferencia pues de las variantes precedentes por su pronunciado carácter higrófilo y terri-humícola.

Su comportamiento esciófilo permite relacionarla sintaxonómicamente con las comunidades nemorales de Fissidens taxifolius, en las que son determinantes diferentes especies humícolas de suelo de bosque (a veces formando sinusia), donde tiene lugar una sucesión de duración variable (cfr. Sjögren, 1964: 49; Willmanns, 1966: 255; Hübschmann, 1975: 304, etc ). Sin embargo, el carácter higrófilo de la va- 
riante nevadense le aproxima más bien a aquellas comunidades de Fissidens taxifolius, cuya presencia está ligada a microclimas húmedos en microestaciones de fuentes, manantiales y pequeños arroyos (cfr. Heinemann \& Vanden Berghen, 1946: 59; Haybach, 1956: 154; Ghesten، \& Wattez, 1971: 72; Dünk, 1972: 41; Mihai, 1973: 413 y 1977: 129). En este sentido, Neumayr (1971: 124, tabl.10, invent. 11-15) reproduce parcialmente nuestra variante. En el territorio que aquí se estudia, es la que marca la transición hacia Brachythecio-Cardaminenion.

\section{Facies de Pellia endivifolia y Thamnobryum alopecurum (tabl.1, invent. 20 y 25-30)}

Pellia endiviifolia y Thamnobryum alopecurum, son considerados diferenciales de una facies basófila y marcadamente esciófila que se desarrolla casi siempre en el seno de la variante higro-esciófila de Plagiomnium undulatum, así como más débilmente en las otras variantes. Sus estaciones participan de unas características comunes: iluminación escasa o nula y sustratos enriquecidos en bases (pH: 77,5), favoreciendo la entrada de un taxon subatlántico y neutrófilo como Thamnobryum alopecurum, junto con los basófilos Pellia endiviifolia, Gymnostomum aeruginosum y Adiantum capillus-veneris, configurando un mosaico de variado significado briosociológico.

Nomenclatura: La comunidad de Fissidens taxifolius ha sido mencionada bajo nombres diversos, recibiendo además un tratamiento descriptivo y sintaxonómico bastante heterogéneo. En la tabla sintética que presentamos (tabl. 2) se reunen los datos proporcionados por diversos autores, procedentes en su mayoría de territorios centroeuropeos y escandinavos.

Las comunidades de dicha tabla muestran ciertas afinidades florísticas, así como ecológicas conforme a las descripciones de sus autores; es por ello que las consideramos expresiones matizadas de una misma comunidad $y$, por lo tanto, sinónimas en sus distintas denominaciones (cfr. Sjögren, 1964:58).

La más antigua, Swartzietum Waldheim 1944, aunque publicada válidamente, requiere sin embargo una corrección nomenclatural según los artículos 14 y $14 \mathrm{~b}$ del "Code of Phytosociological Nomenclature"; conforme indica el artículo 39, la denominación correcta sería la de Fissidentetum taxifolii Waldheim 1944 que es utilizada por Marstaller (1980:99) para una comunidad que tiene como características a Fissidens taxifolius y Eurhynchium hians, asimismo presentes en la descripción original de Waldheim.

Por sus afinidades ecológicas y brioflorísticas, la comunidad de Fissidens taxifolius de Sierra Nevada la incluimos en la asociación Fissidentetum taxifolii Waldheim 1944. Sin embargo, por razones biogeográficas, la comunidad nevadense se expresa mediante la subasociación rhizomnietosum punctati que posee peculiaridades diferenciales respecto de las comunidades descritas de centroeuropa y Escandinavia, como las que siguen:

La ausencia de Eurhynchium hians (=E. swartzii), una de las especies diferenciales en la subasociación typicum, que es poco frecuente en el sur de la Península.

Como contraste, es de notar en nuestra comunidad la fuerte presencia de Eurhynchium praelongum y Rhizomnium purctatum, en relación con las comunidades descritas para centroeuropa y Escandinavia.

Por su carácter mesohigrófilo y microclimático. la comunidad 
Tabla 1 FISSIDENTETUM TAXIFOLII Waldheim 1944 RHIZOMNIETOSUM PUNCTATI subas. nova

\begin{tabular}{|c|c|c|c|c|c|c|c|c|c|c|c|c|c|}
\hline Númerc de orden & 1 & 2 & 3 & 4 & 5 & 6 & 7 & 8 & 9 & 10 & 11 & 12 & 13 \\
\hline Superficie $\left(\mathrm{dm}^{2}\right)$ & 2 & 15 & 12 & 9 & 20 & 11 & 12 & 8 & 20 & 20 & 12 & 10 & 10 \\
\hline Cobertura (\%) & 70 & 100 & 50 & 60 & 60 & 80 & 75 & 60 & 60 & 55 & 60 & 60 & 100 \\
\hline Exposición & S & 0 & s & S & $\mathrm{N}$ & $\mathrm{E}$ & $\mathrm{N}$ & $\mathrm{NE}$ & $\mathrm{N}$ & $\mathrm{N}$ & $\mathrm{N}$ & $\mathrm{S}$ & $\mathrm{N}$ \\
\hline Inclinación $\left({ }^{\circ}\right)$ & 90 & 90 & 50 & 90 & 45 & 90 & 90 & 80 & 40 & 10 & 80 & 10 & 70 \\
\hline Sustrato & M & M & M & M & M & $\mathrm{M}_{1}$ & M & M & M & M & M & $M$ & M \\
\hline ilúmero de especies & 8 & 8 & 6 & 6 & 6 & 5 & 5 & 5 & 6 & 7 & 4 & 5 & 8 \\
\hline
\end{tabular}

var. typicum

Característica de asociación (Fissidentetum taxifolii):

Fissidens taxifolius

$+3+333341+31+$

Diferencial territorial:

Eurhynchium praelongum

Diferencial de subasociación (rhizomnietosum punctati):

Rhizomnium punctatum

Diferenciales de variantes:

Amphidium mougeottii

Plagiomnium undulatum

Diferenciales de facies:

Pellia endivifolia

Thamnobryum alopecurum

Especies de Pogonato-Dicranelletea:

Pellia epiphylia

Plagiochila asplenioides

Atrichum undulatum

Especies de Montio-Cardaminetea:

Bryum pseudotriquetrum

Chiloscyphus polyanthos

Philonotis fontana

Cardamine sylvatica

Brachythecium rivulare

Cratoneuron commutatum

$$
\text { var. falcatum }
$$

Compañeras :

Didymodon insulanus

Scapania undulata

Gymnostomum aeruginosum

Adiantum capillus-veneris

Calliergonella cuspidata 


$\begin{array}{rrrrrrrrrrrrrrrrr}14 & 15 & 16 & 17 & 18 & 19 & 20 & 21 & 22 & 23 & 24 & 25 & 26 & 27 & 28 & 29 & 30 \\ 20 & 30 & 100 & 100 & 6 & 80 & 40 & 16 & 4 & 4 & 4 & 9 & 6 & 9 & 9 & 6 & 4 \\ 70 & 60 & 65 & 100 & 80 & 80 & 100 & 60 & 60 & 85 & 100 & 100 & 80 & 100 & 90 & 60 & 60 \\ \mathrm{~N} & \mathrm{NE} & \mathrm{N} & \mathrm{NE} & \mathrm{O} & \mathrm{NE} & \mathrm{E} & \mathrm{NE} & \mathrm{E} & \mathrm{E} & \mathrm{E} & \mathrm{E} & \mathrm{E} & \mathrm{E} & \mathrm{E} & \mathrm{E} & 0 \\ 90 & 90 & 90 & 90 & 90 & 90 & 90 & 90 & 30 & 20 & 90 & 90 & 90 & 90 & 90 & 90 & 90 \\ \mathrm{M} & \mathrm{M} & \mathrm{M} & \mathrm{M} & \mathrm{M} & \mathrm{M} & \mathrm{M} & \mathrm{M} & \mathrm{M} & \mathrm{M} & \mathrm{M} & \mathrm{M} & \mathrm{M} & \mathrm{M} & \mathrm{M} & \mathrm{M} & \mathrm{M} \\ .0 & 11 & 8 & 14 & 9 & 7 & 5 & 8 & 7 & 6 & 7 & 6 & 6 & 6 & 7 & 7 & 6\end{array}$

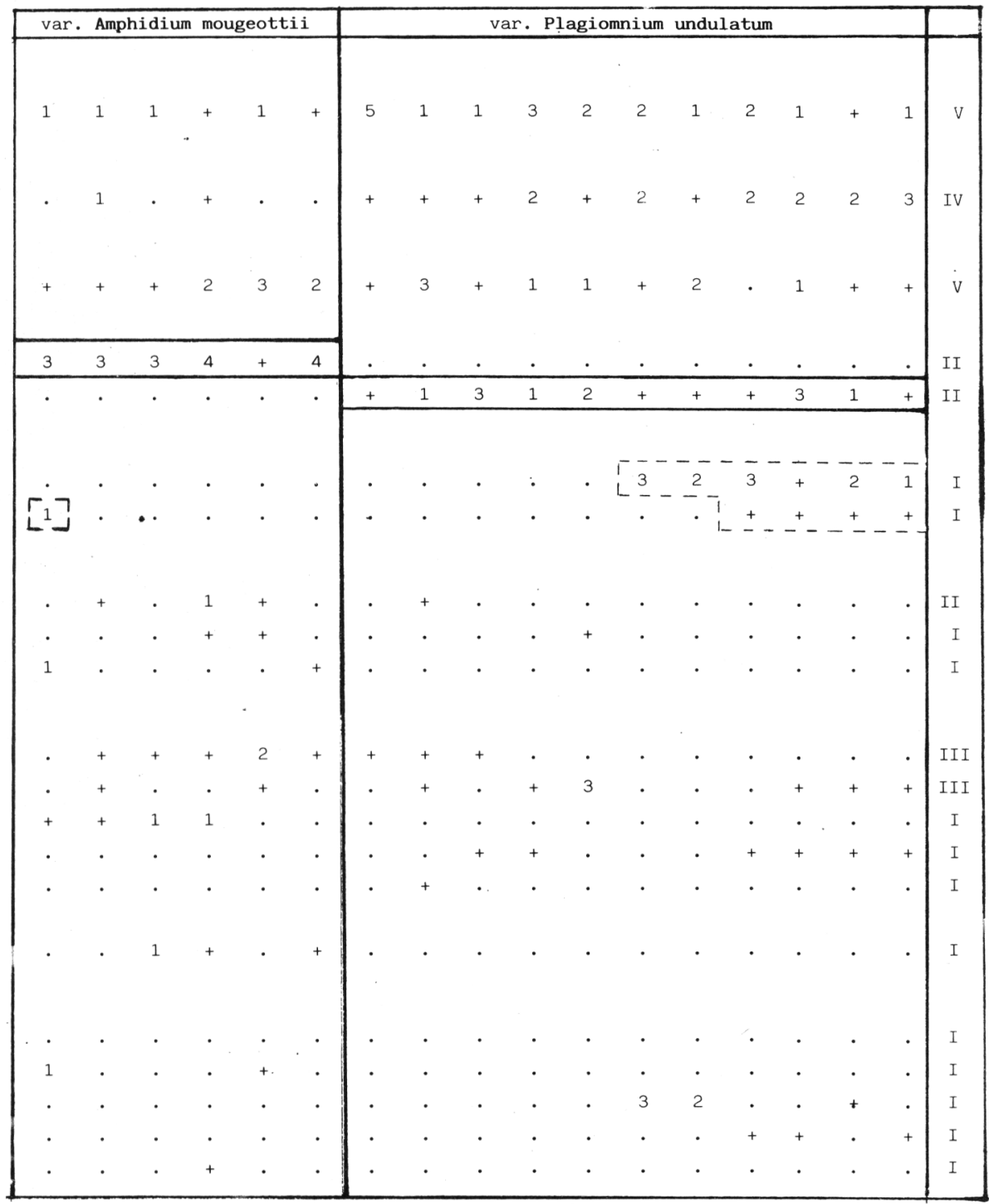

Origen de los inventarios:

1: VG9707 Puerto de la Ragua, 2000 m; 2: VG7716 Dehesa del Camarate, 1400 m; 3, 4,5, 7,9,10,20 y 22: VG 9705 Barranco del Hornillo, 1900m; 6,12,13,30 y 31: VG8610 Bco. río Pueblo, 1550 m; 8: VG9407 Bco. río Pasillo, $1700 \mathrm{~m} ; 23$ y 25: VG9803 Bco. próximo al cruce a Bayárcal, $1800 \mathrm{~m}$; 21: VG9502 Bco. río Laroles, 1900 -m; 24: VF8899 Bco rí Yégen, $1700 \mathrm{~m} ;$ 28: VG8310 Bco. río Alcázar, 1550 m; 29: VG8211 Bco. río Alhorí, $1600 \mathrm{~m}$; 32 VF5992 Bco. río Lạjarón, $1750 \mathrm{~m} ; 33,34,35,36,37$ y 38: VF7288 Fuente Agria de Pórtugos, 
nevadense adquiere su máxima originalidad a partir de un grupo de especies helo-higrófilas que consideramos introgresivas, como se ha dicho, de Brachythecio-Cardaminenion.

Relación con unidades superiores: Algunas comunidades de Fissidens taxifolius han sido descritas como asociación Fissidentetum taxifolii Heinemann \& Vanden Berghen 1946 (sinónima de Swartzietum Waldheim 1944) (cfr. Neumayr, 1971; Dünk, 1972; Mihai, 1973 y 1977; Hübschmann, 1975), siendo articulada indistintamente por unos y otros en Dicranelletalia heteromallaePhilippi 1956 como en Barbuletalia unguiculatae Hübschmann 1967. La tabla 2 ofrece un espectro briofloristico integrado en su mayoría por elementos circumpolares que son indiferentes a la reacción del sustrato. En consecuencia, ninguno de ellos tiene exclusiva relación sintaxonómica con Pogonato-Dicranelletea heteromallae Hübschmann 1973, mesófila y acidófila, ni con Barbuletea unguiculatae Hübschmann 1967, xero-basófila y de óptimo mediterráneo.

De otra parte, estos elementos circun-neutrófilos fueron estructurados por Waldheim (1944), Krusenstjerna (1945), Sjögren (1964) y Willmanns (1966), como unión Swartzietum en la federación Eurhynchion striatae Waldheim 1944, la cual forma en Escandinavia una sinusia nemoral típica de bosques frondosos y eútrofos, bien en taludes $y$ bordes de caminos $o$ en el suelo del sotobosque. Fissidens taxifolius es una de las especies designadas comocaracterística de esta federación, junto a diversas especies de Eurhynchium y Mnium (s.1.). En paises más meridionales, es evidente que este tipo de formaciones adquieren distinto significado, teniendo que refugiarse en biótopos frescos o húmedos próximos a algún cauce.

Según el estado actual de conocimientos sobre las comunidades briofíticas terrícolas en Europa, y sobre todo en la Región Mediterránea, estos nos parecen no sólamente heterogéneos sino además insuficientes como para relacionar sintaxonómicamente la asociación, con los sucesivos esquemas propuestos por Waldheim (1944 y 1947), Krusenstjerna (1945), Philippi (1956 y 1965), Sjögren (1964), Neumayr (1971) y Hübschmann (1975). Por otra parte, teniendo en cuenta la ausencia casi total de inventarios de esta comunidad en la Región Mediterránea y del carácter limitado de nuestro trabajo, no nos hallamos en situación de proponer la creación de unidades superiores que puedan recogerla adecuadamente.

Sincorología: La asociación parece extenderse en areales circumboreales de toda Europa, en general supeditada al ambiente nemoral de caducifolios, adquiriendo carácter montano en territorios meridionales. En la Península Ibérica, de momento sólo la hemos observado en microclimas protegidos del piso supramediterráneo nevadense, si bien es probable su existencia en diversas provincias corológicas peninsulares de clima atlántico.

\section{CONCLUSIONES}

La asociación Fissidentetum taxifolii Waldheim 1944 rhizomnietosum punctati subas. nova tiene en Sierra Nevada la misma posición bioclimática y significado ecológico que las formaciones boscosas de Quercus pyrenaica. Por consiguiente, se desarrolla en estaciones microclimáticas que reciben un aporte hídrico extra en verano, independientemente de las precipitaciones. Es por ello que, tanto los robledales como la asociación briofítica que comentamos, se ubican en el fondo de los barrancos, extendiéndose sobre todo en los que se situan en las laderas septentrionales, donde es mayor la humedad rela- 
Tabla 2 TABLA SINTETICA DE LAS COMUNidAdES DE FISSIDENS TAXIFOlius Hedw.

\begin{tabular}{|c|c|c|c|c|c|c|c|c|c|c|c|c|c|c|c|c|c|c|c|}
\hline Número de orden & 1 & 2 & 3 & 4 & 5 & 6 & 7 & 8 & 9 & 10 & 11 & 12 & 13 & 14 & 15 & 16 & 17 & 18 & 19 \\
\hline Número de inventarios & 15 & 7 & 15 & 19 & 8 & 6 & 27 & 5 & . & 2 & 10 & . & . & . & 2 & . & 10 & . & 30 \\
\hline Fissidens taxifolius & $v$ & v & $\mathrm{v}$ & II & IV & II & III & 5 & $\mathrm{x}$ & 2 & I & $x$ & I & $x$ & 2 & $\mathrm{x}$ & $\mathrm{v}$ & $\mathrm{x}$ & v \\
\hline Eurhynchium hians (*) & v & v & I & IV & $\mathrm{v}$ & III & II & 2 & $\mathrm{x}$ & 1 & IV & $x$ & . & . & . & . & . & . & . \\
\hline Fissidens bryoides & $\cdot$ & . & . & . & . & - & . & $\cdot$ & . & . & I & $x$ & $\mathrm{~V}$ & $\mathrm{x}$ & . & . & . & - & . \\
\hline Plagiomnium rostratum & . & III & II & II & II & IV & I & . & . & . & I & . & . & . & 1 & $\mathrm{x}$ & . & . & I \\
\hline Plagiomnium undulatum & $\cdot$ & II & II & I & . & IV & . & 1 & . & . & . & . & II & . & . & $\mathrm{x}$ & - & $x$ & I \\
\hline Brachythecium rutabulum & IV & . & II & I & I & II & . & . & $x$ & . & I & . & . & . & 2 & . & . & $x$ & I \\
\hline Atrichum undulatum & II & I & . & I & . & . & I & 2 & . & . & II & $x$ & . & $x$ & . & . & . & $\mathrm{x}$ & I \\
\hline Plagiochila asplenioides & . & I & III & . & . & . & . & . & $\mathrm{x}$ & . & . & . & v & . & . & $\mathrm{x}$ & II & . & I \\
\hline Eurhynchium striatum & $\cdot$ & . & . & $\cdot$ & . & . & · & $\cdot$ & $\mathrm{x}$ & . & $\cdot$ & . & II & $\mathrm{x}$ & . & $\mathrm{x}$ & I & $x$ & $\cdot$ \\
\hline Amblystegium serpens & . & . & . & II & III & I & . & . & . & . & I & . & IV & . & 1 & $\mathrm{x}$ & . & . & . \\
\hline Barbula unguiculata & I & . & III & . & I & I & . & 1 & . & . & I & . & $\cdot$ & . & . & . & I & . & . \\
\hline Rhizomnium punctatum & . & . & II & •II & . & I & II & . & . & 1 & . & . & . & . & . & . & . & . & $I I I$ \\
\hline Pellia endivifolia & . & . & I & . & II & I & . & 5 & . & . & . & . & . & . & . & $\mathrm{x}$ & - & . & I \\
\hline Lophocolea bidentata & . & . & I & . & . & . & . & 5 & . & . & $\cdot$ & . & II & . & . & $\mathrm{x}$ & II & . & . \\
\hline Brachythecium velutinum & II & . & . & IV & II & . & . & . & . & . & IV & $x$ & . & . & . & . & - & . & . \\
\hline Eurhynchium praelongum & . & . & . & . & . & . & . & 1 & . & . & . & . & $\cdot$ & $x$ & . & . & $\cdot$ & . & IV \\
\hline Dicranella heteromalla & . & . & I & . & . & . & . & 1 & . & . & . & . & . & . & . & . & . & . & . \\
\hline Bryum pseudotriquetrum & $\cdot$ & . & . & . & . & . & . & . & . & . & . & . & $\cdot$ & · & . & $\cdot$ & $\cdot$ & $\cdot$ & III \\
\hline Chiloscyphus polyanthos & $\cdot$ & . & . & · & . & $\cdot$ & · & . & $\cdot$ & . & $\cdot$ & $\cdot$ & $\cdot$ & $\cdot$ & . & $\cdot$ & $\cdot$ & $\cdot$ & III \\
\hline Pellia epiphylla & $\cdot$ & . & . & . & . & - & . & . & . & . & $\cdot$ & $\cdot$ & $\cdot$ & $\cdot$ & · & $\cdot$ & $\cdot$ & $\cdot$ & II \\
\hline Philonotis fontana & $\cdot$ & · & · & · & · & $\cdot$ & $\cdot$ & $\cdot$ & $\cdot$ & · & $\cdot$ & $\cdot$ & $\cdot$ & $\cdot$ & . & $\cdot$ & $\cdot$ & $\cdot$ & I \\
\hline Brachythecium rivulare & $\cdot$ & . & . & . & . & $\cdot$ & . & . & . & . & $\cdot$ & . & $\cdot$ & $\cdot$ & . & . & $\cdot$ & $\cdot$ & I \\
\hline
\end{tabular}

Sintáxones y localidades:

1. Swartzietum Waldheim 1944, tabl. 4: Suecia.

2. Swartzietum Waldheim 1944 (in Sjögren, 1964: 53, tabl.8, invent. 1-7: 0land, Suecia.

3. Fissidentetu taxifolii (Haybach 1956) Philippi 1965 (in Neumayr, 1971: 124, tabl.10: Frankenalb, Alemania).

4. Swartzietum-Ass. Waldheim 1944 (in Mihai, 1973: 413, tabl.1: Uricani, Rumania).

5. Eurhynchietun swartzii Waldheim 1944 (in Mihai, 1977: 127, tabl.I: Iasi, Rumania)

6. Briocenoza Mniun undulatun \& Mniun longirostre Mihai 1976 (in Mihai, 1977:128, tabl. II: Iasi, Rumania).

7. Fissidens taxifolius-Eurhynchiun speciosum-Verein Haybach 1956: 159, tabl.5: Viena, Austria.

8. Swartzietun Waldheim 1944 (in Willmanns, 1966: 256, tabl.4, invent. 10-14: Spitzbergs, Alemania).

9. Fissidentetu taxifolii Heinemann $\&$ Vanden Berghen 1946 (in Hübschmann, 1975: 304: Weser, Alemania).

10. Swartzietum Waldheim 1944 (in Marstaller, 1973: 580, tabl.24: Thüringen, Alemania).

11. Eurhynchietu swartzii Waldheim 1944 (in Mihai, 1976: 661, tabl.1: Iasi, Rumania).

12. Fissidentetu taxifolii Waldheim 1944 (in Marstaller, 1980: 99: Jena, Alemania).

13. Fissidens bryoides-Ges. Philippi 1965 (in Dünk, 1972: 42, tabl.42: Oberfranken, Alemania.

14. Fissidens taxifolius-Eurhynchiun speciosum-Verein Haybach 1956 (in Ghestem \& Wattez, 1971: 72: Haute-Marche, Bélgica).

15. Fissidentetu taxifolii Heinemann \& Vanden Berghen 1946 (in Mihai, 1977: 128, tabl.II: Iasi, Rumania).

16. Fissidentetu taxifolii Heinemann \& Vanden Berghen 1946 (in Dünk, 1972: 41, tabl.42: Oberfranken, Alemania).

17. Fissidens taxifolius-Ges. Philippi 1965: 654, tabl.18: Wutach, Alemania).

18. Fissidentetum taxifolii Heinemann \& Vanden Berghen 1946: 59: Anlier, Bélgica.

19. Fissidentetum taxifolii Waldheim 1944 rhizomnietosum punctati subas. nova (Sierra Nevada, España). 
tiva como consecuencia de una menor insolación. Otro factor microclimático que incide igualmente en el desarrollo lo constituye la inversión de temperaturas, por la acumulación de aire frío y por tanto más denso, en el fondo de los barrancos.

La relación existente entre esta comunidad briofítica de Sierra Nevada y los robledales, es semejante a la que tienen otras comunidades de Fissidens taxifolius descritas para diversos territorios centroeuropeos y escandinavos, asimismo ligadas al bioclima de caducifolios (fundamentalmente roble), como sinusia o comunidad nemoral de taludes y suelo de sotobosque.

Sin embargo, los melojares béticos han reducido progresivamente su distribución desde el Cuaternario, como consecuencia de un clima cada vez más adverso, restringiéndose su área a estaciones microclimáticas favorables. En este sentido, los táxones briofíticos eurosiberianos han experimentado el mismo proceso, y por ende la asociación briofítica de Sierra Nevada, que se diferencia por su carácter higrófilo más acentuado.

En definitiva, al igual que los melojares béticos de AdenocarpoQuercetum pyrenaicae, la Fissidentetum taxifolii rhizomnietosum punctati se caracteriza por su posición finícola y relíctica respecto a las comunidades descritas en territorios de la Europa central y septentrional.

\section{BIBL IOGRAF IA}

APINIS, A. \& LACIS, L. -1936- Data on the ecology of bryophytes. II. Acidity of the substrate of Musci. Acta Horti Bot. Univ. Latv., 9-10: $1-95$.

BARKMAN, J.J., MORAVEC, J. \& RAUSCHERT, S. -1976- Code of Phytosociological Nomenclature. Vegetatio, 32(3): 131-185.

CASAS, C. -1981- The mosses of Spain: An annotated Check-List. Treb. Inst. Bot. Barcelona, 7:1-56.

DUNK, K.v. -1972- Moosgesellschaften im Bereich des Sandsteinkeupers in Mittel- und Oberfranken. Ber. Naturwiss. Ges. Bayreuth, 14: 7-100.

GHESTEM, A. \& WATTEZ, J.R. -1971- Aperçu sur la végétation bryophytique d'un secteur de la Haute-Marche. Bull. Soc. Bot. N. France, 24:6184.

GROLLE, R. -1976- Verzeichnis der Lebermoose Europas und benachbarter Gebiete. Feddes Repert., 87(3-4): 171-279.

HAYBACH, G.-1956- Zur Okologie und soziologie einiger Moose und Moosgesellschaften des nordwestlichen Wienerwaldes. Verhandl. Zool. Bot. Ges. in wien, 96: 132-168.

HEBRARD, J.P. -1973- Contribution à l'étude de quelques bryoassociations de l'étage subalpin dans le sud-est de la France. Vegetatio, 27(4$6): 347-381$.

HEINEMANN, P. \& VANDEN BERGHEN, C. -1946- Aperçu sur la végétation bryophytique de la fôret d'Anlier. Bull. Soc. Roy. Belgique, 78:55-64.

HUBSCHMANN, A.v. -1975- Moosgesellschaften des Nordwestdeutschen Tieflandes Zwischen Ems und Weser. II. Teil: Erdmoosgesellschaften. Heŕzogia, 3: 275-326.

KRUSENSTJERNA, A.E.v. -1945- Bladmossvegetation och Bladmossflora i Uppsala-Trakten. Acta Phytogeogr. Suec., 19: 1-250.

MARSTALLER, R. -1973- Die Bryophytenvegetation des Naturschutzgebietes "Waldecker Schlossgrund" (Kreis Stadtroda, Thüringens). Wiss. Z. Univ. Jena, math.-nat. R., 22: 545-590.

MARSTALLER, R. -1980- Die Moosgesellschaften des Verbandes Phascion mitriformis Waldheim 1947. 7. Beitrag zur Moosvegetation Thüringens. Feddes Repert., 91: 363-387. 
MIHAI, G. -1973- Bryologische Untersuchungen aus dem Wald Uricani-Iassi. An. st. Univ. "Al. I. Cuza" din Iassi. S.N., 19(2): 411-420.

MIHAI, G. -1976- Microassociations muscinale de la Fôret de Mîrzesti. Feddes Repert., 87(9-10): 659-669.

MIHAI, G. -1977- Cercetari asupra vegetatiei muscinale higro-si hidrofile din Masivul Paduros Birnova-Repedea (Iasi). St. si Cerc. Biol. seria Biol. Veget., 29(2): 137-141.

NEUMAYR, L. -1971- Moosgesellschaften der sudöstlichen Frankenalb und des Vorderen Bayerischen Waldes. Hoppea Denkschr. Regensburg. Bot. Ges., $29(1 / 2): 1-364$.

NORR, M. -1969- Die Moosvegetation des Naturschutzgebietes Bodetal. Hercynia, 7(1/3): 13-52.

OCHSNER, F. -1975- Die Moosflora der montanen Stufe des Raumes RamoschStrada und der angrenzenden Gebiete des Unterengadins. Ergeb. Wiss. Untersuch. Schweiz. Nationalpark, 12: 213-285.

PHILIPPI, G. -1956- Einige Moosgesellschaften des Südschwarzwaldes und der angrenzenden Rheinebe. Beitr. Naturk. Forsch. Suidwestdeutschl., 15: $91-124$.

PHILIPPI, G. -1965- Die Moosgesellschaften der Wutachschlucht. Mitt. bad. Landesver. Naturkd. Natursch., N.F. 8(4): 625-668.

SJOGREN, E. -1964- Epilithische und epigäische Moosvegetation in Laubwäldern der insel Oland (Schweden). Acta Phytogeogr. Suecica, 48: 1148 .

WALDHEIM, S. -1944- Mossvegetation i Dalby-Söderskogs Nationalpark. Kgl. Svensk. Vetensk. Akad. Avh. Natturskyddsär, 4: 1-142.

WALDHEIM, S. -1947- Kleinmoosgesellschaften und Bodenverhältnisse in Schonen. Bot. Notis., Suppl.1: 1-203.

WILLMANNS, O. -1966- Die Flechten- und Moosvegetation des Spitzbergs. (In :Der Spitzberg bei Tübingen). Natur. und. Landschaftsschutzgeb. Baden-Württemb., 3: 244-277. 